\section{Macular ischaemia in Behçet's disease}

\author{
Abstract \\ Purpose To report macular ischaemia and \\ visual loss in patients with panuveitis due to \\ Behçet's disease. \\ Methods We describe macular ischaemia, a \\ rare finding, in 3 eyes of 3 patients with \\ panuveitis who were diagnosed and treated as \\ having Behçet's disease. The patients \\ underwent fundus fluorescein angiography \\ (FFA) using a digital imaging system and were \\ treated with topical and oral steroids and \\ cyclosporine in 2 cases, and with added \\ azathioprine in the third case. \\ Results The 3 eyes showed macular ischaemia \\ associated with peripheral retinal vasculitis on \\ FFA and control of inflammation was achieved \\ in all cases. After an average of 2 years \\ follow-up, visual acuity and macular \\ ischaemia improved in 2 eyes, while no \\ recovery was seen in the third. \\ Conclusion The presence of macular \\ ischaemia on FFA in Behçet's disease is a \\ predictor of poor visual outcome. Macular \\ ischaemia may show partial recovery with the \\ treatment of the disease.
}

Key words Behçet's disease, FFA, Macular ischemia, Panuveitis, Vasculitis

Behçet's disease is a chronic multisystem disorder characterised by a generalised vasculitis of unknown aetiology. In 1990, the International Study Group for Behçet's Disease (ISGBD) proposed a standardised set of diagnostic criteria. ${ }^{1}$ These include recurrent oral ulceration and two of the following findings: characteristic eye lesions, recurrent genital ulceration, characteristic skin lesions, or a positive pathergy test. The ocular manifestations of Behçet's disease include nongranulomatous iridocyclitis, occlusive retinal vasculitis involving both arteries and veins, inflammatory response of the vitreous, retinal haemorrhages, infiltrates and oedema. ${ }^{2}$ The posterior segment involvement leads to irreversible alterations and serious visual loss. Although macular alterations, such as macular oedema, macular hole, epiretinal membrane formation and subretinal neovascularisation ${ }^{3}$ secondary to Behçet's disease and other uveitis have been reported, macular ischaemia ${ }^{4,5}$ has been noted in just a few reports. In this report
GÜRSEL YILMAZ, YONCA AKOVA, PINAR AYDIN

we discuss the findings on fundus fluorescein angiography (FFA) of the presence of macular ischaemia in 3 eyes of 3 patients with active panuveitis and Behçet's disease.

\section{Case reports}

Case 1

A 30-year-old man was referred to us for blurred vision, redness and pain in the right eye and mild visual loss in the left eye. On initial examination, best corrected visual acuity was counting fingers at $3 \mathrm{~m}$ in the right eye, 20/20 in the left eye. Slit-lamp examination revealed $2+$ cells in the anterior chamber and $3+$ cells in the vitreous in the right eye, and $1+$ cells in the anterior chamber and $1+$ cells in the vitreous in the left eye. Fundus examination showed cystoid macular oedema, retinal vasculitis and retinal haemorrhages in the right eye, and mild cystoid macular oedema in the left eye. FFA showed leakage from the optic disc, irregularity of the foveal avascular zone and perifoveal capillary drop-out with microvascular abnormalities (Fig. 1a,b), and retinal vasculitis in the right eye. Hyperfluorescence of the optic disc and cystoid macular oedema were seen in the left eye. Behçet's disease was diagnosed based on the ISGBD criteria since our patient had a history of erythema-nodosum-like eruptions, recurrent oral aphthous ulcerations, genital ulceration and arthritis. In addition, HLA typing showed that the HLA B51 antigen was present and a pathergy test was positive.

Topical, transseptal and systemic corticosteroids as well as oral cyclosporin A (300 mg/day) treatment was initiated. With this therapy, the inflammation was controlled with resolution of vitreous inflammation and retinal infiltrates. Visual acuity improved to 20/200 within 3 weeks and we observed that macular ischaemia partially resolved on FFA (Fig. 1c). Both the visual acuity and macula remained stable during the 2 year follow-up period under treatment.

\section{Case 2}

A 19-year-old man presented complaining of blurred vision, redness and pain in both eyes, with the left eye worse than the right. The patient reporting having had blurring of vision for the previous 4 months in both eyes. He had a
G. Yilmaz
Y. Akova

P. Aydin

Başkent University School of Medicine

Department of

Ophthalmology

Ankara, Turkey

Dr Gürsel Yılmaz

Başkent University School of

Medicine

Department of

Ophthalmology

Bahçelievler, 06490

Ankara, Turkey

Tel: +90 3124359408

Fax: +90 3122237333

e-mail:

gurselyilmaz@hotmail.com

Received: 7 February 2000 Accepted in revised form: 12 May 2000 


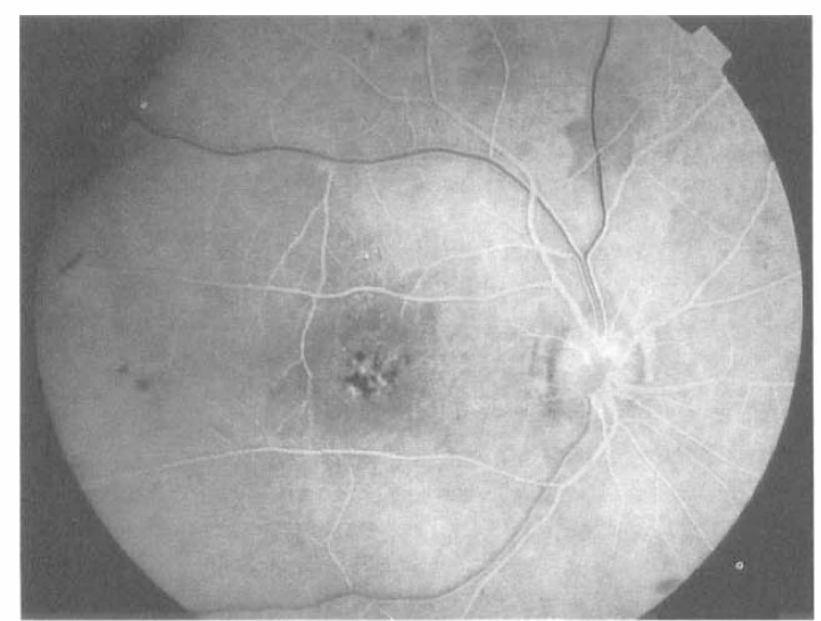

(a)

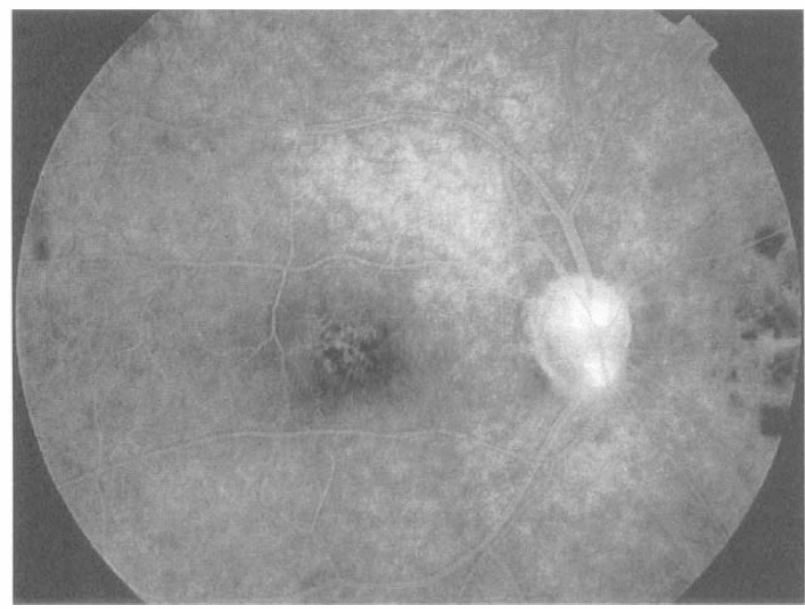

(c)

history of genital ulceration, oral aphthous lesions, arthritis and erythema-nodosum-like eruptions. On examination, best corrected visual acuity was 20/20 in the right eye and 20/200 in the left. Slit-lamp examination revealed mild conjunctival hyperaemia, $1+$ cells in the anterior chamber and $1+$ cells in the vitreous in the right eye; and conjunctival hyperaemia, fine keratic precipitates, $3+$ cells in the anterior chamber, posterior synechiae and $3+$ cells in the vitreous in the left eye.

Fundus examination showed cystoid macular oedema in both eyes. Additionally, retinal vasculitis, haemorrhages and infiltrates were present in the left eye. FFA demonstrated optic disc staining and cystoid macular oedema in the right eye, leakage from the optic disc, irregularity of the foveal avascular zone and macular ischaemia (Fig. 2a) with retinal vasculitis in the left eye. Pathergy test was positive.

The patient was diagnosed with Behçet's disease according to ISGBD criteria and treated with topical, transseptal and systemic corticosteroids and oral cyclosporin A. With this therapeutic regime inflammation was controlled, the visual acuity improved to 20/60 in the left eye within 3 months and the macular ischaemia partially resolved on FFA (Fig. 2b). Although

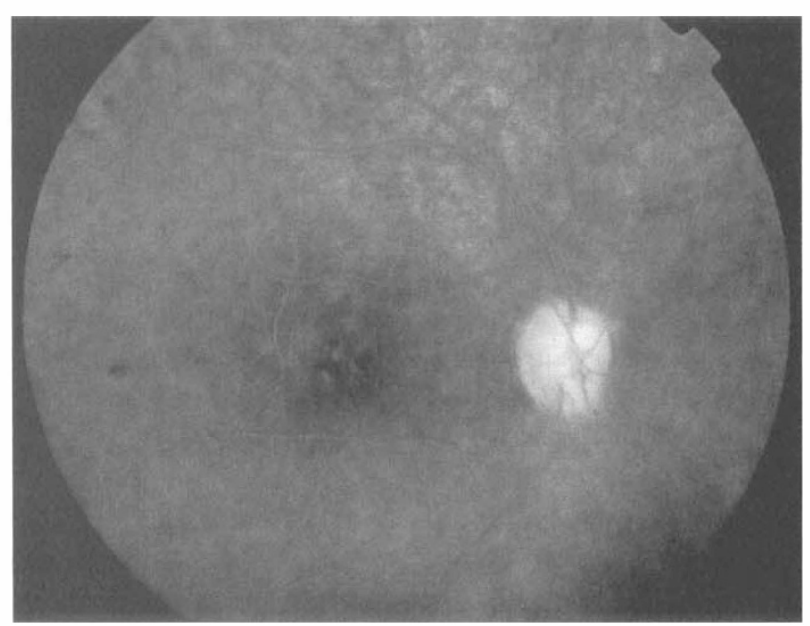

(b)

Fig. 1. Case 1. (a) Fundus fluorescein angiography (FFA) demonstrating perifoveal capillary drop-out with microvascular abnormalities in the right eye before treatment. (b) FFA showing leakage from the optic disc in the right eye in the late phase. (c) Macular ischaemia partially resolved after treatment.

he had several mild flare-ups within this time, macular findings and visual acuity in both eyes remained the same in the 2 year follow-up period.

\section{Case 3}

A 50-year-old man applied to the hospital's outpatient clinic complaining of bilateral visual loss. He had been treated for Behçet's disease and followed for the previous 8 years at another institution, and was referred for further evaluation. He was given oral cyclosporine therapy at a dosage of $350 \mathrm{mg} /$ day. Cataract surgery had been performed on the right eye 2 years previously. On examination visual acuity was $20 / 400$ in the right eye and 20/200 in the left. Slit-lamp examination showed $1+$ cells in the anterior chamber, pseudophakia and $1+$ cells in the vitreous in the right eye; and $1+$ cells in the anterior chamber, nuclear cataract and $3+$ cells in the vitreous in the left eye.

Fundus examination revealed marked attenuation of the retinal vasculature, sheathing of the vessels, and cystoid macular oedema in both eyes. Mild disc pallor was also noted in both eyes. FFA showed hyperfluorescence of the optic disc, vasculitis in the right eye and cystoid macular oedema, retinal vasculitis and 


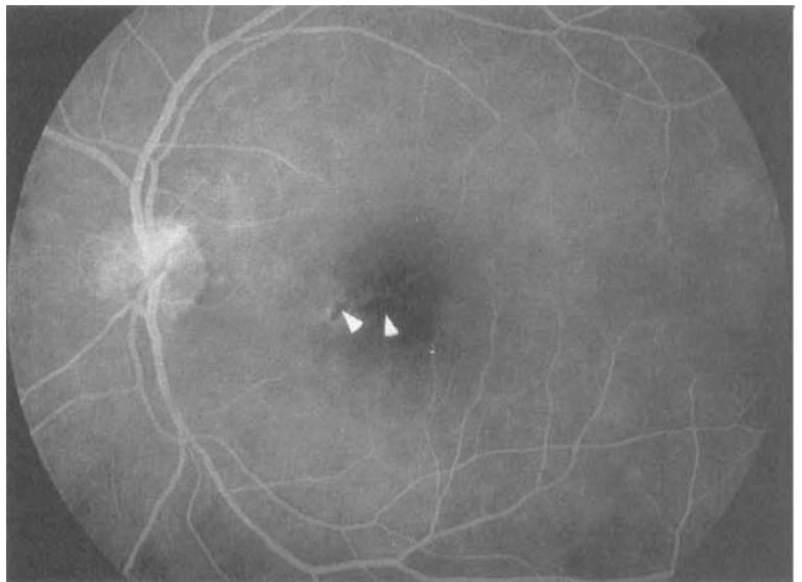

(a)

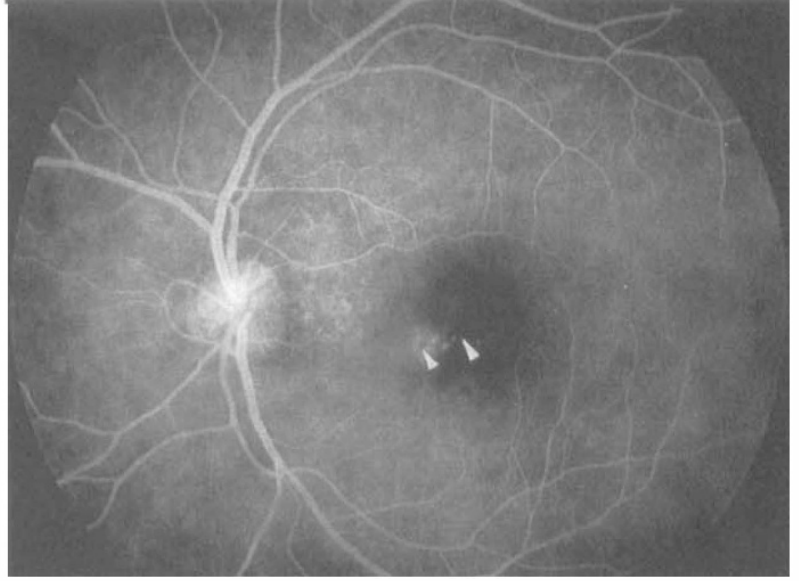

(b)

Fig. 2. Case 2. (a) Fundus fluorescein angiography showing leakage from the optic disc, irregularity of the foveal avascular zone and macular ischaemia (arrowheads) with retinal vasculitis in the left eye before treatment. (b) Macular ischaemia (arrowheads) partially resolved after treatment.

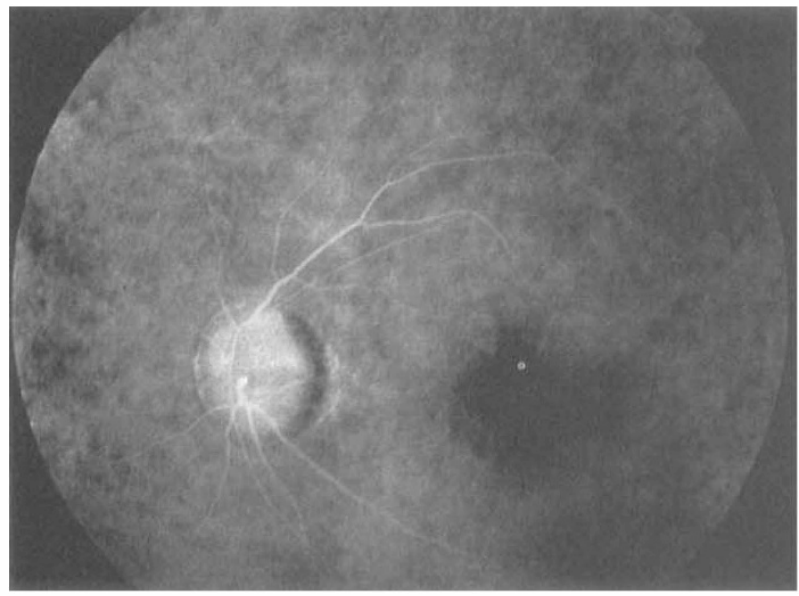

(a)

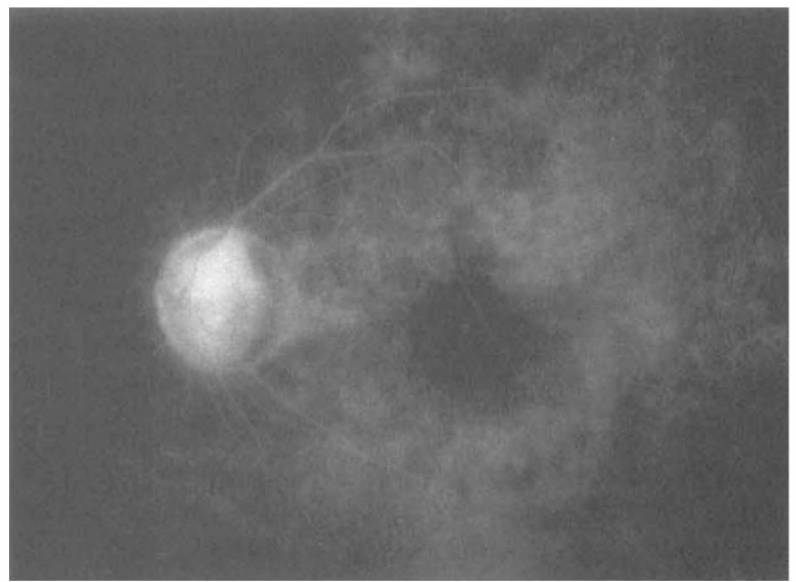

(b)

Fig. 3. Case 3. (a) Fundus fluorescein angiography demonstrating hyperfluorescence of the disc, retinal vasculitis and irregularity of the foveal avascular zone. (b) Macular ischaemia did not improve despite treatment.

irregularity of the foveal avascular zone in the left eye (Fig. 3a). Systemic signs included oral ulceration, genital ulcers, erythema nodosum and arthritis.

The diagnosis of Behçet's disease was confirmed and azathioprine (150 mg/day) was added to the treatment regime with systemic steroids. Visual acuity did not improve and macular ischaemia did not resolve on FFA (Fig. 3b). Six months later, cataract surgery was performed on the left eye without any complication. Although inflammation was under control, neither the macular ischaemia nor the visual acuity improved during the 2 year follow-up period.

\section{Discussion}

Ocular involvement ranges from $70 \%$ to $85 \%$ in patients with Behçet's disease. Although the hallmark of Behçet's disease is the presence of panuveitis and vasculitis, the sequelae of the inflammation appear to be sightthreatening. Visual loss is the most serious complication arising from Behçet's disease and results from longstanding inflammation and consequent macular oedema, retinal and optic nerve atrophy and, in some cases, retinal vascular occlusions that cause ischaemic retinopathy with neovascularisation, vitreous haemorrhage or neovascular glaucoma. Although all macular aspects of visual morbidity have been reported, macular ischaemia has not been well documented. Garcher et al. ${ }^{4}$ have reported a case of bilateral visual loss and macular ischaemia related to Behçet's disease and Bentley et al. ${ }^{5}$ have reported macular ischaemia in 12 patients with posterior uveitis, 4 of whom were diagnosed with Behçet's disease.

The cause of macular ischaemia is thought to be occlusive retinal vasculitis, but in Behçet's disease retinal microvascular occlusions usually occur in larger vessels than the perifoveal capillaries. Vasculitis, characterised by both a perivascular and intramural infiltrate of lymphomononuclear cells, is the common histopathological mechanism in all organ systems affected by Behçet's disease. This vasculitis can lead to vascular obliteration and fibrinoid necrosis of tissue. George and co-workers ${ }^{6}$ demonstrated histopathology of an eye revealing marked non-granulomatous uveitis 
with a predominantly CD4+ T-lymphocytic infiltration, as well as B-cell and plasma cell aggregation. In addition, the vascular endothelium showed extensive expression of adhesion molecules and major histocompatibility class II antigens. They concluded that both cell-mediated immunity and immune-complex deposition were responsible for the pathophysiology of Behçet's disease. On the basis of these findings, one may speculate that macular ischaemia seen in Behcet's disease may be due to immune-complex deposition in the perifoveal capillaries, together with cellular and humoral immunological mechanisms and adhesion molecules. Furthermore, patients with Behçet's disease have elevated levels of anticardiolipin antibodies, high fibrinogen levels and high viscosity in the active stage., Additionally, factor $\mathrm{V}$ Leiden may be an additional risk factor for the development of retinal vaso-occlusion, ${ }^{9}$ but haematological and clotting screens were normal in all our patients.

Systemic steroids, cytotoxic agents, colchicine and cyclosporine are the most commonly used agents in the treatment of Behçet's disease. However, even though they reduce the frequency and severity of inflammatory attacks, these drugs have no effect on either retinal capillary non-perfusion or optic disc and retinal neovascularisation. As far as macular ischaemia is concerned, Garcher et al. ${ }^{4}$ reported a case of recovery of macular ischaemia and visual acuity with high-dose corticosteroid treatment. However, visual acaity failed to improve in 4 patients who had macular ischaemia with Behçet's disease despite treatment with prednisolone and cyclosporin A or azathioprine in another study. ${ }^{5}$ In our cases, 2 of 3 patients improved in terms of both visual acuity and macular ischaemia while the remaining patient showed no improvement in either visual acuity or recovery of macular ischaemia.

Retinal involvement is the most disquieting aspect of Behçet's disease and can ultimately lead to severe visual loss. ${ }^{10,11}$ We consider active panuveitis as the treatment guideline in our patients. However, when we achieved control of the inflammation, the macular ischaemia also improved in 2 of 3 patients. Little attention has been paid to macular ischaemia in the past; it is a rare finding in Behçet's disease and there are no established criteria concerning its treatment in these patients. However, in our experiences described above, when inflammation is brought under control, macular ischaemia may also improve. Every effort should be made to control ocular inflammation in Behçet's disease.

\section{References}

1. International Study Group for Behçet's Disease. Criteria for diagnosis of Behçet's disease. Lancet 1990;35:1078-80.

2. Michelson JB, Chisari FV. Behçet's disease. Surv Ophthalmol 1982;26:190-203.

3. Nussenblatt RB. Macular alterations secondary to intraocular inflammatory disease. Ophthalmology 1986;93:984-8.

4. Garcher C, Bielefeld P, Desvaux C, Besancenot JF, Bron A. Bilateral loss of vision and macular ischemia related to Behçet's disease. Am J Ophthalmol 1997;124:116-7.

5. Bentley CR, Stanford MR, Shilling JS, Sanders MD, Graham EM. Macular ischaemia in posterior uveitis. Eye 1993;7:411-4.

6. George MRK, Chan CC, Whitcup SM, Nussenblatt RB. Ocular immunopathology of Behçet's disease. Surv Ophthalmol 1997;42:157-62.

7. Hull RG, Harris EN, Gharavi AE, et al. Anticardiolipin antibodies: occurrence in Behçet's syndrome. Ann Rheum Dis 1984;43:746-8.

8. Hampton KK, Chamberlain MA, Menon DK, Davies JA. Coagulation and fibrinolytic activity in Behçet's disease. Thromb Haemost 1991;66:292-4.

9. Verity DH, Vaughan RW, Madanat W, et al. Factor V Leiden mutation is associated with ocular involvement in Behçet's disease. Am J Ophthalmol 1999;128:352-6.

10. Akova YA, Yilmaz G, Aydin P. Retinal tears associated with panuveitis and Behçet's disease. Ophthalmic Surg Lasers 1999;30:762-5.

11. Nussenblatt RB, Whitcup SM, Palestine AG. Uveitis: fundamentals and clinical practice. 2 nd ed. St Louis: $\mathrm{CV}$ Mosby, 1996:334-53. 